OPEN ACCESS

Edited by:

Alvin H. Schmaier,

Case Western Reserve University,

USA

Reviewed by:

Owen McCarty,

Oregon Health \& Science University,

Keith $R$

University of Washington, USA

*Correspondence:

Meral Beksac

beksac@medicine.ankara.edu.tr

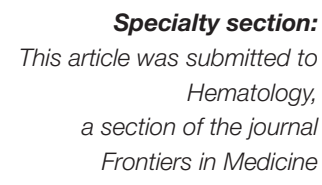

Received: 31 October 2015

Accepted: 18 December 2015

Published: 11 January 2016

Citation:

Beksac M (2016) Is There Any

Reason to Prefer Cord Blood Instead of Adult Donors for Hematopoietic

Stem Cell Transplants?

Front. Med. 2:95.

doi: 10.3389/fmed.2015.00095

\section{Is There Any Reason to Prefer Cord Blood Instead of Adult Donors for Hematopoietic Stem Cell Transplants?}

\author{
Meral Beksac* \\ Ankara University, Ankara, Turkey
}

As cord blood (CB) enables rapid access and tolerance to HLA mismatches, a number of unrelated CB transplants have reached 30,000. Such transplant activity has been the result of international accreditation programs maintaining highly qualified cord blood units (CBUs) reaching more than 600,000 CBUs stored worldwide. Efforts to increase stem cell content or engraftment rate of the graft by ex vivo expansion, modulation by molecules such as fucose, prostaglandin E2 derivative, complement CD26 inhibitors, or CXCR4/CXCL12 axis have been able to accelerate engraftment speed and rate. Furthermore, introduction of reduced intensity conditioning protocols, better HLA matching, and recognition of the importance of HLA-C have improved CB transplants success by decreasing transplant-related mortality. CB progenitor/stem cell content has been compared with adult stem cells revealing higher long-term repopulating capacity compared to bone marrow-mesenchymal stromal cells and lesser oncogenic potential than progenitor-induced stem cells. This chapter summarizes the advantages and disadvantages of CB compared to adult stem cells within the context of stem cell biology and transplantation.

\section{Keywords: stem cell transplantation, engraftment, donor matching}

Among many sources of hematopoietic stem cell sources, cord blood (CB) is the one that belongs to the earliest stages of life. Thus, $\mathrm{CB}$ has many features resembling fetal or even embryonic stem cells. This review will focus on biological features of $\mathrm{CB}$ and comparison of transplants with $\mathrm{CB}$ vs. adult stem cells.

\section{CORD BLOOD STORAGE WORLDWIDE}

More than three million units of $\mathrm{CB}$ are currently stored worldwide for autologous or altruistic purposes. Most recent bone marrow donors worldwide (BMDW) website shows that there are 676,446 unrelated CB units (CBUs) stored in 43 registries. This number is continuously increasing. Although CBUs belong to $2.5 \%$ of the stem cell pool, thanks to introduction of $\mathrm{dCB}$ transplants (dCBT) that contribute to approximately $20 \%$ of the unrelated stem cell transplants $(1,2)$. The usual odds ratio is 1 out of 100 CBUs that meet donor-recipient eligibility/match criteria and are transplanted. For a long time, low stem cell content was the major limiting factor against more frequent usage. The previous experience among pediatric patients have now expanded to adults, and the number of $\mathrm{CB}$ transplants has reached 30,000. Allogeneic unrelated CB transplants dominate the annual CBT statistics.

Cord blood units, unlike adult donor stem cell collection/harvests, are readily available on demand. Confirmatory HLA Typing and organization of transportation are the few barriers prior 
to CBT. Hence, CB offers an advantage for patients in need of urgent transplantation. As CBTs can be performed with a 4-6/6 match, this presents a chance of transplantation for patients who possess rare HLA types, i.e., who belong to ethnical groups not well-represented in registries or who belong to different race admixture families.

The HLA data of CBUs in the registries have improved in accordance with the required level and extend of typing as for the unrelated adult donors. CB banks are encouraged to perform collection, transportation, processing, and storage according to the standards published and audited by international authorities (FACT/NETCORD or AABB). The main purpose is to maintain high quality of stem cell products. Post-thaw stem cell counts are now being considered as the most important determinant of a successful engraftment (3). Purtill et al. have recently shown that CBUs from accredited banks engraft better.

\section{AMENDMENT OF MATCHING CRITERIA FOR CORD BLOOD}

The matching criteria for unrelated CBUs, until very recently, were based on low/intermediate HLA-A,-B, and high-resolution HLADRB1 with a minimum match level of $\geq 4 / 6$. In 2011, the EBMT and CIBMTR have published the importance of HLA-C typing. Compared to $8 / 8$ matches, those mismatched at HLA-C had a higher transplant-related mortality (TRM) (HR: 3.97, $p=0.018)$. A 5/6 mismatch plus a HLA-C mismatch also increased the TRM (HR: $1.7, p=0.029$ ) (4).

Until recently, with the exception of high-resolution HLADRB1 typing, CBUs were typed at low-resolution level for HLA-A and -B loci. Recently, Oran et al. (5) have shown better high-resolution HLA-A-, HLA-B-, HLA-C-, and -DRB1-matched cords to determine the dominating $\mathrm{CBU}$ following double $\mathrm{CB}$ transplants. Additionally, they did not observe any 2-year transplant-related mortality among 7-8/8-matched patients. With increasing number of mismatches, TRM increased as well. Hence, better matching at a four digit level and four loci increases the success rate.

Although it may seem controversial, the Minnesota group recently published allele level mismatching based on the predominant unit to be only important for prevention of relapse (HR: $3.4, p=0.01$ ) (6). Otherwise, allele level mismatching was not associated with any significant detrimental effect on TRM, engraftment, or GVHD. In this study, there were only 32 out of 342 patients who were $9-10 / 10$ allele matched. Although there was a reduction in TRM, it was not significant. One additional explanation for the difference between these two analyses could be due to comparison being performed at five or four loci. The Minnesota group proposes to aim for higher cell content units to overcome the potential problems that will arise from HLA mismatches. They argue a more selective approach trying to match CBUs at allelic level will decrease the number of CBTs. However, the New York Group has shown that by using a model in which both a higher eight-allele HLA match and a cell dose $\geq 2.0 \times 10^{7} / \mathrm{kg} /$ unit were required, graft selection changed in $33 \%$ of transplants with minimal effect on cell dose (8.3\% reduction). They conclude that while units chosen based on HLA-A, -B antigen, and -DRB1 allele match have substantial mismatch at higher resolution, and $\mathrm{CB}$ selection based on high-resolution HLA match is possible in a significant proportion of patients without compromising cell dose $(7,8)$.

The acceptability of HLA mismatches within the CBT setting can also be explained by matching for non-inherited parental HLA alleles (9). During pregnancy, fetal lymphocytes may be exposed to maternal antigens (NIMA) and gain tolerance. The maternal haplotype, which is not inherited can only be recognized by maternal HLA typing. NIMA matching has been shown to decrease graft-vs.-host disease (GVHD). CB Registries have now initiated the inclusion of maternal HLA types. In the case of a NIMA match, the $4 / 6$ match can increase to a virtual 5/6 match grade. As a result, Europdonor and National Cord Blood program have shown that the elucidation of donors' maternal HLA phenotypes can provide significant numbers of $6 / 6$ and $5 / 6$ virtually matched CBUs to patients and is potentially costeffective (10).

In conclusion, recent data favor HLA-C, NIMA, and highresolution typing to influence outcome and thus are recommended to be introduced into donor selection criteria. Although the role of HLA-C has been ascertained extensively, the impact of high-resolution typing and of which locus/loci remains to be investigated (6).

The number of HSCs transplanted in a CBT protocol is at least one log less than a PBSCT or BMT. On the other hand, these cells are cryopreserved and post-thaw viability may range between 75 and $99 \%$. The outcome of CBT is affected negatively if recipient has developed anti-erythrocyte antibodies or alloimmunization prior to transplantation. In such situations, to avoid engraftment failure, the best HLA match and highest CD34 dose are required. However, the New York Group has published data negating the effects of allo-immunization $(7,8)$. Although an insignificant delay in median number of days for neutrophil engraftment ( 23 vs. 31 days) was observed, they have hypothesized that using an immunosupressive conditioning, lack of ATG and double unit grafts abrogates the deleterious effects of anti-HLA antibodies.

\section{MODIFICATION OF DOUBLE CORD BLOOD TRANSPLANT PROTOCOLS}

To increase immunosupression as a tool to overcome HLA mismatch effects, either fludarabine or ATG has been added to the conventional myeloablative conditioning (MAC) regimens. As summarized by Ruggeri et al. in the Eurocord/EBMT study, two MAC regimens were equally effective (11). The Fludara, Busulphan, and Thiotepa regimen (single CBT) or Fludara, Cyclophosphamide, and TBI regimens (dCBT) were the MAC protocols available widely. The Minnesota Group was the first to introduce non-myeloablative approach in CBT (12). The New York Group modified this regimen using cyclophosphamide (50 $\mathrm{mg} / \mathrm{kg})$, fludarabine $\left(150 \mathrm{mg} / \mathrm{m}^{2}\right)$, thiotepa $(10 \mathrm{mg} / \mathrm{kg})$, and 400 cGy total body irradiation with cyclosporine-A/mycophenolate mofetil immunosuppression without ATG. As a result they were able to achieve engraftment at a median of 26 days among 
$97 \%$ (13). Platelets recovered among $93 \%$ by day 180 . Grades II-IV acute GVHD incidence was $67 \%$ at day 180 , and chronic GVHD was $10 \%$ at 1 year. TRM was $20 \%$ at day 180 , and relapse was $11 \%$ at 2 years. Overall, 2-year disease-free survival (DFS) was $60 \%$ at 2 years. Likewise, Kai et al. have reported results with a myeloablative regimen of CyTBI or CyTBI plus high-dose of cytosine arabinoside (14). This study once again proved the importance of HLA matching on engraftment. Furthermore, standard risk patients obtained a plateau of $67 \%$ event-free survival at 3 years compared to $29 \%$ among advanced disease patients $(p=0.023)$. In conclusion, introduction of NMA or RIC regimens have improved engraftment and EFS (40 vs. $60 \%)(6,13)$.

\section{CORD BLOOD AS A SOURCE OF STEM CELLS}

Umblical arterial CB vessels transport blood from the fetal hepatic circulation to the placental feto-maternal interface to be replaced with clean fresh blood devoid of metabolites. Umblical venous blood contains fetal cells, which have been in contact with placental intervillous syncytothrophoblasts. Oxygen and nutrient transport is maintained at this anatomical border throughout pregnancy. Placenta is an organ where cells of the fetus come into very close proximity but do not meet directly with maternal cells. The structure of placenta allows contact of fetal syncytothrophoblasts with maternal blood cells or decidua cells called interstitial trophoblasts. Successful pregnancy is maintained with suppression of immune reactivity either by lack of surface HLA expression, secretion of IGF-1/2, LIF, or TGF. This highly immunosuppressive environment contributes to the metabolomic profile of $\mathrm{CB}$.

For many years, $\mathrm{CB}$ has been used for hematopoietic stem cell transplantation only. Recently, CB mesenchymal stromal cells (MSCs) have become clinically available. There is an estimated frequency of 1,000-5,000 MSCs in a typical UCB unit of approximately $100 \mathrm{~mL}$ (15). The main advantage of using CB-MSC is to enhance engraftment and to prevent or to treat GVHD. MSCs can be obtained from multiple sources, i.e., bone marrow, adipose or dental tissues, CB or Wharton's jelly and even menstrual blood.

\section{COMPARISON OF MSC FROM DIFFERENT SOURCES}

Comparison of MSCs obtained from different sources has been a topic of interest for many years. The decline in MSC number and differentiation potential with increasing age is a disadvantage against adult tissues, such as BM or adipose tissues. A comparison by Kern et al. was able to find no significant differences concerning the morphology and immune phenotype of the MSCs derived from different sources. However, differences could be observed concerning the success rate of isolating MSCs, which was $100 \%$ for BM and AT, but only $63 \%$ for UCB. The colony frequency was lowest in UCB, whereas it was highest in AT. However, UCB-MSCs possessed the longest culture period and showed the highest proliferation capacity, BM-MSCs possessed the shortest culture period and the lowest proliferation capacity. In a more recent study by Trivanović et al., in addition to immuno-phenotyping, proliferation and differentiation gene expression assays were also performed. Peripheral blood MSCs (PB-MSCs) and UC-MSCs showed the highest, while periodontal ligament MSCs (PDLMSCs) and adipose tissue MSCs (AT-MSCs) the lowest values of both the replication potential and Relative Telomere Length. Although MSCs from exfoliated deciduous teeth (SHEDs), PDL-MSCs, and AT-MSCs showed higher mRNA expression of pluripotency markers, all MSCs expressed pluripotency marker proteins (16).

These two studies have shown that UC-MSCs have the highest proliferation rate and transplantation capacity than other MSCs $(16,17)$. If the origin is Wharton Jelly, MSCs are cultured efficiently because these cells are maintained in an early embryologic phase and therefore have retained some of the primitive stemness property resulting with a high proliferative capacity (18).

\section{COMPARISON OF CB VS. IPS}

One argument against storage of $\mathrm{CB}$ has arisen following successful generation of induced progenitor cells (IPSC). As these IPSCs have features similar to embryonic stem cells and can be obtained from adult tissues, they can be used in the same individual in need and from whom they were obtained. Currently, there are major obstacles against their routine use in clinical transplantation: their safety has not yet been proven and the production is very expensive and limited to advanced sites only.

Recently, a study comparing the MSC from CB vs. IPSC has shown that the CB MSC's expression level of pluripotent genes OCT4 and Sox-2, Nanog and lin28 were significantly higher than those in IPSCs. In contrast, the expression level of oncogenic factors c-Myc and Klf4 were significantly higher in IPSCs than in CB-MSC. In conclusion, the authors suggested that CB-MSC is may be less oncogenic than IPSC. (19)

\section{BENEFICIAL IMMUNOLOGICAL FEATURES OF CORD BLOOD}

The typical feature of CB lymphocytes is as follows: the majority consists of antigen-inexperienced naïve lymphocytes, which are less responsive to allo-stimulation with a low effector cytokine production capacity. Furthermore, regulatory T cells (T regs) are seen more frequently. UCB dendritic cells have a lower antigen presenting activity. All these features are protective against GVHD but may predispose to graft failure or delay in immune reconstitution $(20,21)$.

On the other hand, UCB is rich in primitive $\mathrm{CD} 16^{-} \mathrm{CD} 56^{++}$ NK cells, which possess impressive proliferative and cytotoxic capacities and can be induced to expand using IL-12 or IL-15, so as to mount a substantial graft-vs.-leukemia (GvL) effect. The composition of $\mathrm{CB}$ mononuclear cells by Tregs and primitive NK cells is an ideal combination serving the purpose of GVL in the setting of allogeneic stem cell transplantation. This topic has been extensively addressed in a chapter in this issue of the journal. 


\section{EX VIVO EXPANSION AND ENGRAFTMENT ENHANCERS}

Unlike all the factors addressed above, methods of CB expansion and engraftment include the most innovative approaches in the field of CBT and CB banking. They are described in detail in the current issue and elsewhere (22, 23, Beksac and Yurdakul, under review for publication in the same Research Topic).

In conclusion, this ready to use stem cell product has many clinically proven indications and protocols. More recent protocols for matching and transplantation have proven to improve transplant success. Furthermore methods under pre- and clinical investigations are very promising toward even better outcomes.

\section{REFERENCES}

1. Barker JN, Byam C, Scaradavou A. How I treat: the selection and acquisition of unrelated cord blood grafts. Blood (2011) 117(8):2332-9. doi:10.1182/ blood-2010-04-280966

2. Wagner JE Jr, Eapen M, Carter S, Wang Y, Schultz KR, Wall DA, et al. One-unit versus two-unit cord-blood transplantation for hematologic cancers. $N \mathrm{Engl} \mathrm{J}$ Med (2014) 371(18):1685-94. doi:10.1056/NEJMoa1405584

3. Purtill D, Smith K, Devlin S, Meagher R, Tonon J, Lubin M, et al. Dominant unit CD34+ cell dose predicts engraftment after double-unit cord blood transplantation and is influenced by bank practice. Blood (2014) 124(19):2905-12. doi:10.1182/blood-2014-03-566216

4. Eapen M, Klein JP, Sanz GF, Spellman S, Ruggeri A, Anasetti C, et al. Effect of donor-recipient HLA matching at HLA A, B, C, and DRB1 on outcomes after umbilical-cord blood transplantation for leukaemia and myelodysplastic syndrome: a retrospective analysis. Lancet Oncol (2011) 12(13):1214-21. doi:10.1016/S1470-2045(11):70260-1

5. Oran B, Cao K, Saliba RM, Rezvani K, de Lima M, Ahmed S, et al. Better allele-level matching improves transplant-related mortality after double cord blood transplantation. Haematologica (2015) 100(10):1361-70. doi:10.3324/ haematol.2015.127787

6. Brunstein CG, Petersdorf EW, DeFor TE, Noreen H, Maurer D, MacMillan ML, et al. Impact of allele-level HLA mismatch on outcomes in recipients of double umbilical cord blood transplantation. Biol Blood Marrow Transplant (2015). doi:10.1016/j.bbmt.2015.09.025

7. Dahi PB, Ponce DM, Devlin S, Evans KL, Lubin M, Gonzales AM, et al. Donorrecipient allele-level HLA matching of unrelated cord blood units reveals high degrees of mismatch and alters graft selection. Bone Marrow Transplant (2014) 49(9):1184-6. doi:10.1038/bmt.2014.135

8. Dahi PB, Barone J, Devlin SM, Byam C, Lubin M, Ponce DM, et al. Sustained donor engraftment in recipients of double-unit cord blood transplantation is possible despite donor-specific human leukoctye antigen antibodies. Biol Blood Marrow Transplant (2014) 20(5):735-9. doi:10.1016/j. bbmt.2014.01.017

9. van Rood JJ, Stevens CE, Smits J, Carrier C, Carpenter C, Scaradavou A. Reexposure of cord blood to noninherited maternal HLA antigens improves transplant outcome in hematological malignancies. Proc Natl Acad Sci U S A (2009) 106(47):19952-7. doi:10.1073/pnas.0910310106

10. Van der Zanden HG, Van Rood JJ, Oudshoorn M, Bakker JN, Melis A, Brand A, et al. Noninherited maternal antigens identify acceptable HLA mismatches: benefit to patients and cost-effectiveness for cord blood banks. Biol Blood Marrow Transplant (2014) 20(11):1791-5. doi:10.1016/j.bbmt.2014.07.011

11. Ruggeri A, Sanz G, Bittencourt H, Sanz J, Rambaldi A, Volt F, et al. Comparison of outcomes after single or double cord blood transplantation in adults with acute leukemia using different types of myeloablative conditioning regimen, a retrospective study on behalf of Eurocord and the Acute Leukemia Working Party of EBMT. Leukemia (2014) 28(4):779-86. doi:10.1038/leu.2013.259

12. Brunstein C, Barker JN, Weisdorf DJ, DeFor TE, Miller JS, Blazar BR, et al. Umbilical cord blood transplantation after nonmyeloablative conditioning: impact on transplantation outcomes in 110 adults with hematologic disease. Blood (2007) 110(8):3064-70. doi:10.1182/blood-2007-04-067215
As one of the pioneers in CBT has said this is a great time for those involved in CB biology and transplantation.

\section{AUTHOR CONTRIBUTIONS}

The author has defined and written the contents of the Research Topic and Mini Review.

\section{FUNDING}

The author acknowledges the research funding provided by Turkish Academy of Sciences in the preparation and publishing of this manuscript.

13. Ponce DM, Sauter C, Devlin S, Lubin M, Gonzales AM, Kernan NA, et al. A novel reduced-intensity conditioning regimen induces a high incidence of sustained donor-derived neutrophil and platelet engraftment after double-unit cord blood transplantation. Biol Blood Marrow Transplant (2013) 19(5):799-803. doi:10.1016/j.bbmt.2013.02.007

14. Kai S, Wake A, Okada M, Kurata M, Atsuta Y, Ishikawa J, et al. Double-unit cord blood transplantation after myeloablative conditioning for patients with hematologic malignancies: a multicenter phase II study in Japan. Biol Blood Marrow Transplant (2013) 19(5):812-9. doi:10.1016/j.bbmt.2013.02.011

15. Rogers I, Casper RF. Umbilical cord blood stem cells. Best Pract Res Clin Obstet Gynaecol (2004) 18:893-908. doi:10.1016/j.bpobgyn.2004.06.004

16. Trivanović D, Jauković A, Popović B, Krstić J, Mojsilović S, Okić-Djordjević I, et al. Mesenchymal stem cells of different origin: comparative evaluation of proliferative capacity, telomere length and pluripotency marker expression. Life Sci (2015) 141:61-73. doi:10.1016/j.lfs.2015.09.019

17. Kern S, Eichler H, Stoeve J, Klüter H, Bieback K. Comparative analysis of mesenchymal stem cells from bone marrow, umbilical cord blood, or adipose tissue. Stem Cells (2006) 24(5):1294-301. doi:10.1634/stemcells.2005-0342

18. Watson N, Divers R, Kedar R, Mehindru A, Mehindru A, Borlongan MC, et al. Discarded Wharton jelly of the human umbilical cord: a viable source for mesenchymal stromal cells. Cytotherapy (2015) 17(1):18-24. doi:10.1016/j. jcyt.2014.08.009

19. Foroutan T, Najmi M, Kazemi N, Hasanlou M, Pedram A. Lower oncogenic potential of human mesenchymal stem cells derived from cord blood compared to induced pluripotent stem cells. Int J Organ Transplant Med (2015) 6(3):99-104.

20. Kim YJ, Broxmeyer HE. Immuneregulatory cells in umblical cord blood and their potential roles in transplantation tolerance. Crit Rev Oncol Hematol (2011) 79(2):112-26. doi:10.1016/j.critrevonc.2010.07.009

21. Kanda J, Chiou LW, Szabolcs P, Sempowski GD, Rizzieri DA, Long GD, et al. Immune recovery in adult patients after myeloablative dual umbilical cord blood, matched sibling, and matched unrelated donor hematopoietic cell transplantation. Biol Blood Marrow Transplant (2012) 18(11):1664.e-76.e. doi:10.1016/j.bbmt.2012.06.005

22. Danby R, Rocha V. Improving engraftment and immune reconstitution in umbilical cord blood transplantation. Front Immunol (2014) 5:68. doi:10.3389/ fimmu.2014.00068

23. Mehta RS, Rezvani K, Olson A, Oran B, Hosing C, Shah N, et al. Novel techniques for ex vivo expansion of cord blood: clinical trials. Front Med (2015) 2:89. doi:10.3389/fmed.2015.00089

Conflict of Interest Statement: The author declares that the research was conducted in the absence of any commercial or financial relationships that could be construed as a potential conflict of interest.

Copyright (C) 2016 Beksac. This is an open-access article distributed under the terms of the Creative Commons Attribution License (CC BY). The use, distribution or reproduction in other forums is permitted, provided the original author(s) or licensor are credited and that the original publication in this journal is cited, in accordance with accepted academic practice. No use, distribution or reproduction is permitted which does not comply with these terms. 